
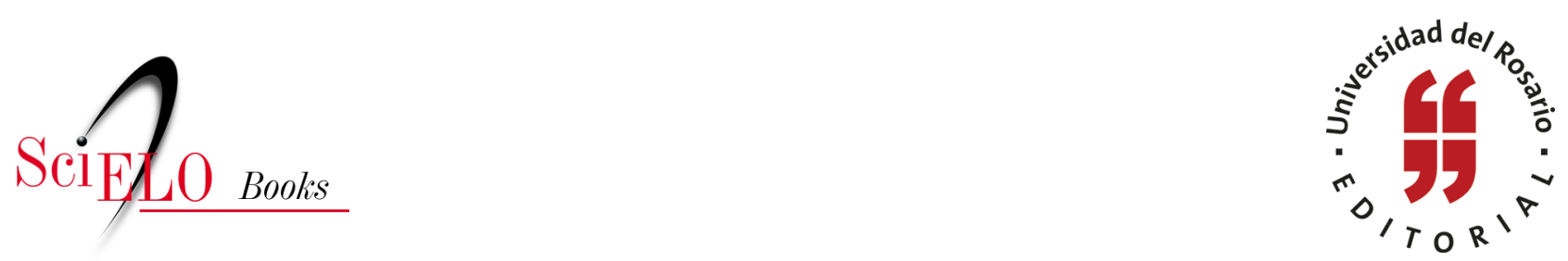

\title{
Capítulo 2. Estratificación socioeconómica en Colombia Contexto general y diagnóstico
}

\author{
Jaime Bonilla \\ Denis López \\ Carlos E. Sepúlveda
}

SciELO Books / SciELO Livros / SciELO Libros

BONILLA, J., LÓPEZ, D., and SEPÚLVEDA, C.E. Estratificación socioeconómica y la información catastral. Introducción al problema y perspectivas a futuro. In: SEPÚLVEDA RICO, C.E., LÓPEZ CAMACHO, D., and GALLEGO ACEVEDO, J.M., eds. Los límites de la estratificación: en busca de alternativas [online]. Bogotá: Editorial Universidad del Rosario: Alcaldía Mayor de Bogotá D.C., 2014, pp. 23-39. ISBN: 978-958-738-537-3. https://doi.org/10.7476/9789587385373.0004.

International license.

Todo o conteúdo deste trabalho, exceto quando houver ressalva, é publicado sob a licença Creative Commons Atribição 4.0.

Todo el contenido de esta obra, excepto donde se indique lo contrario, está bajo licencia de la licencia Creative Commons Reconocimento 4.0. 


\title{
Capítulo 2 \\ Estratificación socioeconómica en Colombia Contexto general y diagnóstico
}

\author{
Jaime Bonilla*, Denis López y Carlos E. Sepúlveda***
}

Palabras clave: avalúo catastral, errores de inclusión y exclusión, solidaridad y redistribución, régimen tarifario, clasificación catastral.

\section{Introducción}

En la aplicación de una política pública en materia de servicios públicos domiciliarios (SPD), Colombia tiene un sistema sui generis de clasificación de los inmuebles residenciales que surge como una aproximación a la capacidad económica de sus residentes. El propósito inicial de dicha clasificación era determinar, en la implementación de una política de focalización de gasto público, los usuarios de SPD que deberían recibir subsidios y aquellos que deberían pagar una contribución de solidaridad dada su mejor situación de ingresos ${ }^{1}$. Este sistema de clasificación es la estratificación socioeconómica

* Consultor independiente.

** Consultor asociado Infométrika, SAS.

*** Profesor principal, Facultad de Economía de la Universidad del Rosario.

1 La intervención estatal en los servicios públicos incluye, para el caso colombiano, la de "establecer un régimen tarifario proporcional para los sectores de bajos ingresos de acuerdo con los preceptos de equidad y solidaridad" (L. 142/94, art. 2.9). 
y a través de ella se agrupa a los inmuebles residenciales hasta en seis grupos o estratos que, en términos generales, reflejan la condición de pertenencia a un grupo social específico ${ }^{2}$.

Con la estratificación, el Estado colombiano ha buscado que los hogares con mejores ingresos asistan económicamente a la población de menores ingresos para acceder, vía tarifas subsidiadas, a los servicios públicos domiciliarios (acueducto y alcantarillado, aseo, energía eléctrica y gas natural) en pos de lograr una cobertura universal.

Además de ser un mecanismo para la asignación de otros subsidios y ayudas a quienes se ubican en los primeros deciles de capacidad de pago (pagos diferenciales en el sistema educativo, diferenciación de tarifas de impuestos a la propiedad, diferenciación de tarifas para pagos al Estado, entre otros), con el paso del tiempo, la estratificación se ha implantado tanto en la vida material como en los imaginarios colectivos como un elemento de diferenciación social y económica, cultural e ideológica de la población.

El esquema de estratificación socioeconómica surge en la década de 1980 y toma impulso y forma a partir de la Ley 142 de 1994. Su metodología se desarrolla ante la ausencia de información técnica, objetiva, veraz y universal de calidad sobre la capacidad de pago de los hogares. A lo largo de estas últimas décadas, una serie de estudios nacionales ha indagado sobre la posibilidad de mejorar la clasificación de los inmuebles, como una herramienta que refleje las condiciones socioeconómicas de los hogares (características de una estratificación ideal), así como la efectividad de la estratificación vigente para recoger esas diferencias (DANE, 2011; Econometría, 2006; DAPD, 2005).

Este capítulo busca brindar una perspectiva general de la estratificación socioeconómica en Colombia. Se desarrolla primero un contexto básico sobre sus propósitos e implementación, y a través de una revisión de literatura,

2 La estratificación fue concebida como un instrumento para determinar subsidios cruzados que partieran del costo de prestación del servicio y subsidios o contribuciones para diferentes grupos de población, atendiendo los principios de solidaridad y redistribución de ingresos. De esta forma, aquellos domicilios habitados por hogares con ingresos inferiores al precio base debían pagar una tarifa menor a este; mientras que quienes percibían un mayor ingreso pagarían un precio superior a ese promedio, considerándose esta como una contribución directa al sistema. Así se pretendía conciliar los criterios de asignación de recursos en la economía, necesidades financieras del sector y distribución de ingresos en el país (Perry, 1983). 
y se presenta un diagnóstico en cuanto a su efectividad en el propósito de focalización de política de subsidios en las tarifas de servicios públicos domiciliarios. Adicionalmente, se hace una revisión sobre estudios que incorporan la información catastral como insumo alternativo para construir modelos de estratificación socioeconómica.

Se destacan las siguientes conclusiones: con el paso de los años, el instrumento de estratificación dejó de reflejar la diferenciación de grupos a partir de su capacidad socioeconómica; el esquema de estratificación presenta errores de inclusión significativos (desde el punto de vista del ingreso, pobreza o capacidad de pago de los hogares) que lleva a una focalización de recursos públicos inadecuada; existe un alto nivel de correlación entre las características de las viviendas, medidas a través de la información catastral (las variables de calificación de construcción y la determinación de zonas homogéneas físicas y geoeconómicas), y las condiciones socioeconómicas de los hogares; la información catastral ofrece así un conjunto importante de variables que permiten caracterizar, diferenciar y agrupar los domicilios usuarios de servicios públicos domiciliarios en grupos homogéneos (estratos) e, incluso, pensar adelante en una política de focalización y manejo de subsidios y contribuciones de SPD soportada directamente en la información catastral, sin necesidad de estratificar.

\subsection{Fundamentos de la estratificación, objetivos y principios}

Atendiendo a principios como la equidad y la solidaridad consagrados en la Constitución, desde los años sesenta, Colombia puso en marcha un mecanismo de fijación de tarifas diferenciales de SPD urbanos en función de "la capacidad económica de los diferentes sectores sociales” (D. 3069/68). Si bien desde los años setenta existió la necesidad de clasificar para asignar tarifas diferenciales, en los años ochenta esta clasificación se hizo explícita a través del inmueble y sus características, generando una cercanía con la información catastral, hasta que el propio marco legal de SPD estableció un régimen propio.

La Ley 142 de 1994 estableció, como pieza angular en el régimen tarifario, la estratificación socioeconómica, como un instrumento para determinar, según la capacidad económica, tanto los subsidios como las contribuciones que 
permitieran aplicar los principios de solidaridad y redistribución de ingresos. Se logra así la implantación de un modelo de subsidios cruzados a la demanda en servicios públicos domiciliarios, cuyo propósito es el de satisfacer los criterios de solidaridad, redistribución de ingresos, simplificación y transparencia, procurando una suficiencia financiera, haciendo que los hogares más pobres paguen una tarifa menor al costo de prestación y que los hogares de mayores ingresos y los sectores industriales y comerciales paguen una sobretasa que permita cubrir los subsidios otorgados. Cuando las contribuciones no son suficientes para financiar los subsidios, el déficit se cubre con los recursos del tesoro nacional, de los presupuestos municipales y de los fondos de solidaridad y redistribución de ingresos.

La estratificación socioeconómica busca responder al propósito de identificación de viviendas objeto de subsidio o contribución, partiendo de dos supuestos básicos. Por un lado, es posible discriminar los usuarios de acuerdo con su nivel de ingresos, capacidad de pago o características socioeconómicas. Y, por el otro, las características del entorno permiten definir grupos homogéneos de usuarios para el cobro diferencial de SPD.

El supuesto de la existencia de estas relaciones condujo a una metodología de estratificación que no considera explícitamente aspectos asociados a las características socioeconómicas de los hogares, sino que realiza la clasificación buscando homogeneidades directamente sobre las características de los inmuebles y su entorno.

La comprensión de los fundamentos y naturaleza de la estratificación socioeconómica parte de entenderla como un mecanismo para atender a una política pública de focalización del gasto público, dirigido a hacer viable el sistema de prestación de SPD en términos financieros, recuperando los costos de prestación del servicio, o parte de ellos, a través de tarifas que atiendan las condiciones económicas de los usuarios de los servicios para otorgar subsidios o cobrar alguna contribución.

Este mecanismo se instrumentaliza a través de la clasificación de los domicilios (inmuebles residenciales) de cada municipio en un máximo de seis estratos (donde el estrato 1 es el más bajo y el 6 el más alto), convirtiéndose al final en un sistema de clasificación predial.

A nivel nacional la metodología de estratificación urbana se materializa en cuatro metodologías, clasificando los municipios en tres tipos, a partir de su 
complejidad económica y social. Para Bogotá, D.C., se diseñó una metodología particular en razón de sus grandes conglomerados de viviendas homogéneas, entre otras particularidades urbanísticas. La estratificación socioeconómica en Bogotá difiere de las metodologías urbanas aplicadas en el resto del territorio nacional, fundamentalmente en tres aspectos:

- Con respecto al nivel de agregación para la asignación del estrato. En Bogotá, se asigna el estrato para el total de la manzana, mientras que, en el resto del país, se asigna el estrato por inmueble residencial en municipios pequeños y por lado de manzana en las ciudades grandes e intermedias.

- Con respecto al conjunto de variables, para Bogotá, se incluye, además de aquellas incorporadas en el resto nacional, variables o conjunto de variables, como, por ejemplo, la 'zona hábitat'.

- Con respecto al método de clasificación, para el caso de Bogotá, se modificó el proceso tradicional utilizado en el resto del país, con el objetivo de tener en cuenta el alto poder de clasificación de la zona hábitat, y se obtuvieron dos dimensiones, lo cual generó la necesidad de aplicar el denominado método de 'Dalenius bivariado', para tener en cuenta las dos variables asociadas a las dimensiones tradicionales y la zona hábitat considerada en Bogotá.

La metodología para Bogotá consta en esencia del siguiente procedimiento (DAPD, 2005): Actualización cartográfica, que consiste en el ajuste de la información espacial, teniendo en cuenta la aparición de nuevas manzanas. Zonificación, que permite establecer el contexto urbanístico de las viviendas a través de la delimitación de zonas hábitat, definidas por aspectos como el uso del suelo, planeación del asentamiento, materiales de construcción, densidad inmobiliaria, estado de culminación del desarrollo, entorno inmediato de la vivienda, calidad del espacio público, grado de deterioro de la edificación y paisaje urbano. Recolección de información por lado de manzana, utilizando un criterio de predominancia de las características evaluadas. 
Tabla 2.1. Variables metodología estratificación vigente

\begin{tabular}{|c|c|c|}
\hline Factores & Variables & Opciones \\
\hline \multirow{29}{*}{$\begin{array}{l}\text { Características de la } \\
\text { vivienda y el entorno }\end{array}$} & \multirow{2}{*}{$\begin{array}{l}\text { V1: Existencia de entrada } \\
\text { principal en las viviendas } \\
\text { del lado de la manzana }\end{array}$} & Sí \\
\hline & & No \\
\hline & \multirow{5}{*}{$\begin{array}{l}\text { V2: Vías de acceso (la calle } \\
\text { o la vía de acceso al lado de } \\
\text { la manzana) }\end{array}$} & Sendero o camino \\
\hline & & Peatonal \\
\hline & & Vehicular en tierra \\
\hline & & Vehicular en recebo basto o gravilla \\
\hline & & Vehicular en cemento, asfalto o adoquín \\
\hline & \multirow{4}{*}{$\begin{array}{l}\text { V3: Tamaño predominante } \\
\text { del frente de las viviendas } \\
\text { del lado de la manzana }\end{array}$} & Hasta 7 metros \\
\hline & & Más de 7 y hasta 9 metros \\
\hline & & Más de 9 y hasta 12 metros \\
\hline & & Más de 12 metros \\
\hline & \multirow{3}{*}{$\begin{array}{l}\text { V4: Andén (predominancia } \\
\text { en el lado de la manzana) }\end{array}$} & Sin andén \\
\hline & & Con andén sin zona verde \\
\hline & & Con andén con zona verde \\
\hline & \multirow{4}{*}{$\begin{array}{l}\text { V5: Antejardín } \\
\text { (predominancia en el lado } \\
\text { de la manzana) }\end{array}$} & Sin antejardín \\
\hline & & Con antejardín pequeño \\
\hline & & Con antejardín mediano \\
\hline & & Con antejardín grande \\
\hline & \multirow{6}{*}{$\begin{array}{l}\text { V6: Garajes (predominancia } \\
\text { en el lado de la manzana) }\end{array}$} & Sin garaje ni parqueadero \\
\hline & & Con garaje cubierto usado para otros fines \\
\hline & & Con parqueadero o zona de parqueo \\
\hline & & Con garaje adicionado a la vivienda \\
\hline & & $\begin{array}{l}\text { Con garaje sencillo que hace parte del diseño original } \\
\text { de la vivienda }\end{array}$ \\
\hline & & Con garajes dobles o en sótano \\
\hline & \multirow{5}{*}{$\begin{array}{l}\text { V7: Material de las fachadas } \\
\text { (predominancia en el lado } \\
\text { de la manzana) }\end{array}$} & En guadua, caña, esterilla, tabla, desechos \\
\hline & & $\begin{array}{l}\text { Sin cubrir - adobe, bahareque, tapia pisada, placa } \\
\text { prefabricada, bloque o ladrillo común }\end{array}$ \\
\hline & & En revoque - pañete o repello - sin pintura \\
\hline & & En revoque - pañete o repello - con pintura \\
\hline & & Con enchapes, en ladrillo pulido o en madera fina \\
\hline
\end{tabular}




\begin{tabular}{|c|c|c|}
\hline Factores & Variables & Opciones \\
\hline \multirow{4}{*}{$\begin{array}{l}\text { Características de la } \\
\text { vivienda y el entorno }\end{array}$} & \multirow{4}{*}{$\begin{array}{l}\text { V8: Material de los techos } \\
\text { (predominancia en el lado } \\
\text { de la manzana) }\end{array}$} & Desechos, telas asfálticas o pedazos de tejas \\
\hline & & Placa de entrepiso \\
\hline & & Terraza, azotea o cubierta sencilla \\
\hline & & Lujosa u ornamental \\
\hline \multirow{14}{*}{ Contexto urbanístico } & \multirow{14}{*}{$\begin{array}{l}\text { Zona por criterios de hábitat } \\
\text { a la cual pertenece la } \\
\text { manzana }\end{array}$} & Zona de pobreza \\
\hline & & Zona de tolerancia \\
\hline & & Desarrollo progresivo sin consolidar \\
\hline & & Deterioro urbanístico \\
\hline & & Industrial \\
\hline & & Desarrollo progresivo consolidado \\
\hline & & Comercial predominante \\
\hline & & Residencial intermedio \\
\hline & & Comercial compatible \\
\hline & & Residencial exclusivo \\
\hline & & Residencial de baja densidad \\
\hline & & Institucional \\
\hline & & Lotes y otros sin vivienda \\
\hline & & Zona verde \\
\hline
\end{tabular}

Fuente: DAPD (2005).

A través del método Savage score ${ }^{3}$, se realiza la transformación de las características de vivienda por predominancia a variables cuantitativas, para la aplicación posterior del método de clasificación. Se consolidan las características de vivienda en una sola variable cuantitativa, quedando dos variables de clasificación: características de vivienda y zona hábitat. Finalmente, se aplica un método de Dalenius-Hodges adaptado (Dalenius bivariado, sobre las dos variables enunciadas) que resulta en la clasificación del conjunto de unidades geográficas en los seis estratos ${ }^{4}$.

3 Para una explicación del método de Savage score, ver el trabajo de Savage (1956) y sus posteriores aplicaciones en R y SAS.

4 Para una revisión detallada del método de Dalenius-Hodge, ver el trabajo de Dalenius y Hodge (1959); para la versión adaptada al caso de Bogotá, ver las Metodologías de estratificación urbana del DANE de 2006. 


\subsubsection{LOS PROBLEMAS DE LA ESTRATIFICACIÓN SOCIOECONÓMICA. DESGASTE PAULATINO DEL INSTRUMENTO}

Diversos estudios de evaluación de la estratificación y su posible relación entre capacidad económica de los hogares y características de los domicilios usuarios de servicios públicos destacan aspectos problemáticos del instrumento de redistribución.

La discusión de cómo aproximarse realmente a las condiciones socioeconómicas de los moradores de los domicilios estratificados ha sido el objeto de distintos estudios y propuestas. Unos intentan establecer la correlación entre ingresos, niveles de gastos y características de las viviendas. Otros correlacionan la capacidad económica de los hogares, medida a partir de indicadores de calidad de vida o de los niveles de pobreza, con la caracterización de las viviendas que ellos habitan. Otros más conectan las características de los domicilios y los entornos a partir de perspectivas multidimensionales de bienestar humano. Más recientemente, otras aproximaciones correlacionan capacidad económica de los hogares según la calidad de vida urbana de que gozan los usuarios de servicios públicos derivada tanto de su nivel económico como del entorno y la calidad de bienes públicos que ofrecen las ciudades. En todos los casos, las aproximaciones coinciden con la perspectiva de la política fiscal que supone que los principios de beneficio y de capacidad de pago hacen referencia a concepciones divergentes de justicia para resolver el problema social de la financiación de los bienes públicos.

Econometría (1993) y Vélez (1996) inician una discusión conceptual y establecen lo que sería una relación ideal entre estratificación e ingreso de los hogares ${ }^{5}$. Sin embargo, al analizar la realidad en la implementación del instrumento, a pesar de presentarse una efectiva correlación positiva, se muestran evidentes problemas de focalización del gasto público en subsidios para tarifas de SPD. Diferentes estudios muestran que, a pesar de que hogares de bajos ingresos se ven efectivamente beneficiados por subsidios en las tarifas de SPD, gran parte de estos recae en hogares de niveles de ingreso o gasto altos.

5 En tal modelo ideal, se asocia el estrato 1 a los dos primeros deciles de ingreso; el estrato 2 a hogares en los deciles 2 al 4; el estrato 3 a los deciles del 4 al 6; el estrato 4 a los deciles del 6 al 8; el estrato 5 a los deciles 8 y 9 ; y el estrato 6 a los deciles 9 y 10 . 
Mina (1997), usando la Encuesta de calidad de vida de 1997 (ECV-97), encuentra disparidades en la distribución de estratos respecto de los deciles de ingreso, aunque evidencia un menor error de inclusión de la estratificación al comparar el estrato con las variables de la ECV. Econometría (1999), a su vez, calcula un modelo para predecir el estrato a partir del ingreso corriente, y encuentra una relación positiva entre los estratos y los ingresos, evidenciando al mismo tiempo una superposición de las funciones de probabilidad para los estratos, dando lugar a una no correspondencia entre los niveles de ingreso y la clasificación de los hogares en estratos distintos al que producen las funciones de probabilidad. Ramírez (2007) ilustra de una manera sencilla este punto a partir de la ECV-03. A nivel nacional, por ejemplo, en el estrato 1, el $30 \%$ de los hogares se encuentra ubicado en los cinco deciles superiores de ingreso, y un 4,2\% de los hogares en este estrato pertenece al decil más alto de ingreso. Estos porcentajes se incrementan para el estrato 3 al 77 y $18 \%$, respectivamente. Estos resultados se confirman en los demás estudios y documentos oficiales (DAPD/CID-UN, 2004; Conpes 3386/05; Econometría, 2006; DANE, 2011; Parra, 2011).

La problemática de la estratificación al estudiar su relación con el ingreso de los hogares se refuerza al tomar como referencia otros indicadores de pobreza o capacidad de pago. Martínez (2004) y DAPD/CID-UN (2004) estudian para Bogotá la relación de los estratos con la incidencia de pobreza por necesidades básicas insatisfechas (NBI), línea de pobreza (LP) y hogares que reciben menos de un salario mínimo per capita. El estudio de DAPD/CID-UN (2004) muestra, además, una correlación con la estructura de consumo de los hogares. Para todos los indicadores, la existencia de errores de inclusión y exclusión es clara. Estratos que reciben subsidios (1, 2 y 3 ) tienen porcentaje significativo de no pobres. Si tomamos, por ejemplo, el estrato 2, el $27 \%$ de los hogares en este grupo no son pobres de acuerdo con LP, y el $22 \%$ de acuerdo con el criterio de un salario mínimo, y aun así reciben subsidios para el pago de tarifas de SPD. Entre tanto, el análisis del gasto corriente es usado para comprobar que el gasto en alimentos y servicios públicos es proporcionalmente mayor en estratos bajos que en los estratos altos, lo que plantea un esquema regresivo en el cobro de tarifas.

Meléndez $(2004,2008)$ propone un índice de focalización de subsidios que también capta la existencia de errores de inclusión y exclusión. Para el caso de acueducto y alcantarillado en Bogotá, se concluye que para 2003 se 
entregaba subsidios a más del $80 \%$ de los hogares no pobres conectados. En términos generales, el estudio afirma que, "a pesar de que la estratificación ha permitido asignar subsidios a los hogares pobres, esto se ha conseguido con el costo de enormes errores de inclusión que se traducen en desperdicio de recursos".

En una evaluación del Departamento Nacional de Planeación (DNP) sobre la estratificación como instrumento de clasificación de usuarios, Econometría (2006) plantea la necesidad de explorar una definición mucho más precisa de la capacidad de pago. Con base en una conceptualización paretiana (teoría del consumidor) propuesta para referir a los niveles mínimos o de subsistencia en el gasto de bienes y el problema de la optimización de este, se analiza un conjunto de variables que conforman un indicador más elaborado de la capacidad de pago para proponer la comparación entre indicadores iniciales de ingreso permanente disponible y el gasto recurrente (que incluye gasto en alimentos y gasto en vivienda) con datos de la Encuesta cocensal del Censo 2005. A partir de ello, se establece la correlación entre la capacidad de pago y las variables socioeconómicas y de pobreza, de una parte; y se busca la explicación que pueda ofrecer la capacidad de pago respecto de la estratificación, de la otra.

Con base en el concepto desarrollado de capacidad de pago, Econometría (2006) analiza la eficiencia de la estratificación tomando como referencia un esquema de clasificación ideal basado en distancias del indicador de capacidad de pago a su media, en unidades de desviaciones estándar. De acuerdo con el estudio, el instrumento presenta un alto nivel de eficiencia y es adecuado para identificar hogares subsidiables, tanto porque subsidia a la gran mayoría de los que tienen menor capacidad de pago $(92,78 \%)$ como porque la gran mayoría de los que no tienen capacidad de pago resultan subsidiados (95,5\%). En la medida en que el monto de subsidio es diferente en el interior de los estratos que realmente los necesitan, la eficiencia se reduce por esta subclasificación y se encuentra que dichos hogares subsidiados no necesariamente se encuentran clasificados en el estrato subsidiable que le corresponde a su capacidad de pago.

No obstante, tal estudio advierte también que el hecho de que el análisis de eficiencia arrojó unos valores muy inferiores cuando no se condiciona a la proporcionalidad entre estratos reales y estratos ideales indica que la estratificación actual ha permitido un crecimiento demasiado alto de los estratos 
subsidiables y ha dejado de incluir a muchos potenciales contribuyentes, lo cual se debería corregir hacia el futuro.

Con base en la revisión de los aspectos más problemáticos que involucra la estratificación en los diagnósticos adelantados en distintos momentos, los estudios sobre la implementación del instrumento evidencian que, con el paso de los años, este dejó de reflejar la desigual capacidad económica aproximada desde los diversos enfoques. Con ello, los errores de exclusión e inclusión se aumentaron poniendo en entredicho la eficacia y efectividad del mecanismo de estratificación. La información base del modelo había perdido vigencia e, incluso, había llegado a ser manipulada por intereses políticos (autoridades que asignaban el estrato según intereses electorales, por ejemplo), o simplemente no había dejado de responder con la suficiente flexibilidad a los cambios ocurridos en la estructura urbana.

Entrado el siglo XXI, era claro para el país que la estratificación de los diez años anteriores resultaba ineficiente por los errores de inclusión y exclusión, por ausencia en el monitoreo de la dinámica socioeconómica y por el uso de la información que alimentaba el modelo de clasificación. Este fue el reconocimiento del documento Conpes 3386 de 2005.

\subsection{De vuelta a la información catastral como fuente de una nueva metodología de estratificación}

Es bueno recordar que, antes de la inclusión de la estratificación socioeconómica, el instrumento que hacía posible la integración de tarifas diferenciales en los esquemas tarifarios era el avalúo catastral. Un ejemplo de esto son las resoluciones de la Junta Nacional de Tarifas en 1981 para el sector residencial en dos municipios de Antioquia: Andes y Argelia, respectivamente.

En este ejemplo, se configuraban categorías en función del avalúo catastral. El número de categorías catastrales variaba de municipio en municipio, pero una mayor variabilidad podía encontrarse en los límites inferiores y superiores de cada categoría. Por ejemplo, en la figura 2.1, la categoría 3 del primer municipio variaba de 30.000 a 70.000 pesos, rango que cubriría las categorías 4, 5 y 6 del segundo municipio. Así mismo, la categoría 5 en el primer municipio inicia en un valor casi cuatro veces superior al que inicia la misma categoría en el segundo municipio. 
1. Acueducto servicio residencial

Para el servicio residencial se establecerán cinco (5) categorías de acuerdo con el avalúo catastral vigente a la fecha de expedición de la presente Resolución. Los cargos fijos mensuales y las tarifas por consumo adicional podrán ser hasta

$\begin{array}{cccc}\text { Avalúo catastral }(\$) & \text { Cargo fijo } & \text { Consumo adicional } \\ \text { Cat. } & \text { Desde } & \text { Hasta } & \text { \$/mes }\end{array}$

Para todas las categorías del servicio residencial tendrán un cupo básico de veinte metros cúbicos $(20$ m3) mensuales.

2. Acueducto servicio residencial

Para el servicio residencial se establecerán seis (6) categorías de acuerdo con el avalúo catastral vigente a la fecha de expedición de la presente Resolución. Los cargos fijos mensuales y las tarifas por consumo adicional podrán ser hasta

\begin{tabular}{|c|c|c|c|c|}
\hline \multicolumn{3}{|c|}{ Avalúo catastral (\$) } & \multirow{2}{*}{$\begin{array}{l}\text { Cargo fijo } \\
\$ / \text { mes }\end{array}$} & \multirow{2}{*}{$\begin{array}{c}\text { Consumo adicional } \\
\$ / \mathrm{M} 3\end{array}$} \\
\hline Cat. & Desde & Hasta & & \\
\hline 1 & 0.000 & 5.000 & 36 & 2.50 \\
\hline 2 & 5.000 & 15.000 & 60 & 3.50 \\
\hline 3 & 15.000 & 25.000 & 80 & 4.50 \\
\hline 4 & 25.000 & 35.000 & 100 & 6.00 \\
\hline 5 & 35.000 & 50.000 & 120 & 7.00 \\
\hline 6 & 50.000 & más & 130 & 7.50 \\
\hline
\end{tabular}

Para todas las categorías del servicio residencial el cupo básico será de hasta veinte metros cúbicos (20 m3) mensuales.

Figura 2.1. Resolución Junta Nacional de Tarifas, JNT004 del 13 de enero de 1981

Fuente: Archivo Comisión de Regulación de Acueducto, Alcantarillado y Saneamiento Básico Ambiental.

La dificultad subyacente a esta opción de clasificación fue la de mantener la información de los avalúos actualizada, de forma tal que estos valores efectivamente reflejaran las condiciones económicas del hogar, sin estar sesgados por decisiones de tipo 'político', como la de mantener artificialmente bajo este valor frente a las realidades del mercado. La variabilidad en los avalúos catastrales se explicaba en parte por la lógica de las decisiones locales de cada municipio, que reducía la posibilidad de que los avalúos catastrales reflejaran 
efectivamente el valor de los predios donde se prestaban los servicios públicos domiciliarios.

Ya en el contexto actual, el documento Conpes 3386 de 2005 parte del diagnóstico del instrumento de estratificación, el cual plantea problemas técnicos, particularmente los errores de inclusión, llevando a trazar lineamientos para la redefinición de su metodología. La dinámica urbana había producido importantes cambios que una metodología debía incorporar para mantener en marcha el sistema cruzado de subsidios y contribuciones en las tarifas de SPD.

Siguiendo una trayectoria abandonada por más de 20 años y tomando como caso de estudio al Distrito Capital, Sardi (2008) reintroduce la utilidad de la información catastral como posible base para la estratificación. A partir del Censo ampliado 2005, por un lado, y las variables contenidas en el sistema catastral, por el otro, muestra que la información catastral ofrece un conjunto de variables que permiten caracterizar y diferenciar los domicilios usuarios de SPD. Sardi (2008) concluye que “... entre la variable estrato actual y las variables de zonas homogéneas económicas y físicas, se evidencia una importante asociación a pesar que la metodología de estratificación adoptada para Bogotá no las considera en forma directa”.

Los resultados de la correlación (canónica no lineal) entre el conjunto de variables de clasificación de los hogares y el conjunto de variables de calificación de las edificaciones y las zonas homogéneas hacen factible utilizar la información catastral como insumo para la estratificación, permitiendo garantizar un blindaje de los resultados que minimice la posibilidad de intervenciones externas que suelen modificar la realidad de las cabeceras municipales y que ponen en riesgo el sistema. Además, la utilización de las variables catastrales garantiza la calidad técnica de la información y la reducción de costos de recolección por los municipios al estar en manos de entidades técnicas definidas para ese propósito, aun con las recomendaciones antes mencionadas, como la actualización de la ficha catastral.

Sin embargo, para ser efectiva, Sardi (2008) recomienda que el IGAC (o la autoridad catastral, según sea el caso) garantice la actualización de las variables de calificación de las edificaciones con uso residencial, en sus definiciones y su asignación a opciones de respuestas que aseguren el carácter ordinal de esta, así como la actualización regular de los modelos econométricos que definen las zonas homogéneas económicas y físicas. 


\subsubsection{HACIA UNA ESTRATIFICACIÓN BASADA EN LA INFORMACIÓN CATASTRAL}

En línea con Sardi (2008), el DANE (2011) realiza un ejercicio de diseño entre 2008 y 2012 que resulta en una propuesta de nueva metodología de estratificación socioeconómica urbana, estudiando la mejor y más actualizada información catastral del país en forma de registros administrativos.

Se retoma de esta manera la posibilidad de utilizar la información catastral como fuente básica y que, al cotejarla con la información censal (particularmente la proveniente de la muestra cocensal) ya disponible, hizo extensivo el análisis para todo el país. Los resultados pusieron en evidencia, de nuevo, el alto nivel de correspondencia entre las características de las viviendas (las variables de calificación de construcción y la determinación de zonas homogéneas físicas y geoeconómicas) y las condiciones socioeconómicas de los hogares.

Los ejercicios realizados por el grupo de diseño de la nueva metodología mostraron que la información catastral constituía un conjunto apropiado, relevante, objetivo y técnico de variables para conformar un modelo de caracterización, clasificación y agrupación de los inmuebles residenciales en todo el país. Igualmente, Maluendas (2013) verificó y aplicó este resultado para el caso especial de Bogotá, D.C.

La propuesta metodológica trabajada por el DANE (2011) incorpora la diferenciación espacial urbana reconociendo que las características constructivas y los niveles de precios de suelo y construcción son variables que captan las diferencias socioeconómicas existentes entre los usuarios de servicios públicos urbanos. Esta relación fue establecida a partir de la asociación existente entre los diferenciales de precios de suelo y construcción en las áreas residenciales, de un lado, y las diferencias en la capacidad económica de los hogares, del otro.

Para su estudio, el DANE basa la construcción en un modelo de clasificación que permite establecer la correspondencia entre variables socioeconómicas y catastrales, a partir de la producción de tres índices que denotan la capacidad de pago esperada de los hogares (el índice de capacidad de pago corriente-ICPc-, el índice de bienestar socioeconómico-IBS- y el índice de distancia al extremo-IDE-), la definición de una muestra de aprendizaje y su clasificación en estratos, todo ello con base en la información (particularmente la proveniente de la muestra cocensal) del Censo general 2005. Posterior- 
mente, se estima un modelo discriminante resultante de la asociación de la clasificación previa a la información catastral (principalmente valor del suelo y calificación de construcción) de los predios urbanos y de centros poblados, clasificados previamente en una tipología que jerarquiza los municipios según su complejidad funcional. Con ello se obtiene la estratificación de inmuebles residenciales a partir de la información catastral, en la que la relación entre condiciones socioeconómicas de los hogares y las características y localización de sus residencias se hace explícita.

La disponibilidad de información, para cada predio individual, contiene una importante ventaja en tanto que la conformación de los estratos socioeconómicos con características comunes y homogéneas puede implementarse a cualquier escala espacial que se requiera (desde las construcciones individuales y los lados de manzana, hasta la manzana, un conjunto de manzanas o cualquier otro dominio espacial).

En interés de la eficiencia y la complementariedad institucional que demandan los principios de la gestión pública, la propuesta metodológica para un nuevo instrumento de estratificación y de acuerdo con la naturaleza de la política de focalización, el uso de los registros catastrales para este propósito es una nueva razón para garantizar la modernización del sistema de información predial. Dicha propuesta metodológica basada en la información catastral debe garantizar los recursos financieros, técnicos y operativos, para lograr una mejor estructuración y actualización de los catastros que permitan al país satisfacer los requerimientos de información técnica no solo para estratificación socioeconómica, sino para los demás fines y funciones del catastro.

En principio, la alimentación de los modelos diseñados por el DANE incluyó variables jurídicas (identificación y detalles de los propietarios, formalidad en la tenencia, etc.), físicas (localización, superficie de terreno y construcción, puntajes de calificación de los diversos ítems relativos a las construcciones y la tipología constructiva) y económicas (valores de las zonas geoeconómicas y valores unitarios y total del suelo y construcción, para inmuebles no pertenecientes al régimen de propiedad horizontal, y valor integral, para aquellos en propiedad horizontal).

El fundamento conceptual de la utilización de la información catastral para la actualización de la metodología de estratificación socioeconómica empleada en el estudio del DANE (2011) se puede sintetizar así: 
1) En el paisaje urbano, las viviendas se construyen con materiales $y$ calidades muy distintas, conforman tamaños dispares y se organizan internamente según las necesidades de los hogares, tienen distinto grado de conservación de sus componentes, y se levantan y desarrollan en entornos y zonas muy diferenciadas geográfica y urbanísticamente.

2) La vivienda urbana evidencia una muy amplia jerarquía de condiciones económicas y sociales de sus residentes, que se expresan en su valor de mercado o en el valor catastral y que son expresión del funcionamiento del mercado de suelo y construcción, expresiones claras de la conformación de rentas diferenciales.

3) La habitabilidad de los distintos tipos de vivienda y su acceso en diversas localizaciones no constituye una elección arbitraria de los moradores. Ella depende ante todo de la disponibilidad por pagar tanto por una determinada vivienda como por una determinada localización intraurbana. En menor medida, influyen en esa decisión la dinámica del mercado inmobiliario, la existencia de patrones sociodemográficos, los comportamientos culturales e, incluso, determinadas conductas subjetivas.

4) La racionalidad de la elección del tipo de vivienda, sus características, su dotación de servicios y su localización relativa en el espacio urbano estriba esencialmente en la capacidad económica, el nivel de ingreso o en la capacidad de pago de las familias. El acceso a la vivienda de ciertas especificaciones constructivas o en una localización geográfica específica está determinado por dicha capacidad.

Desde una aproximación intuitiva y empírica, la construcción residencial, sus características y su entorno constituyen aspectos fácilmente observables y clasificables; y, si se usan criterios objetivos, su caracterización y clasificación en grupos de cierta homogeneidad ofrece transparencia (por ser un elemento relativamente estable en el tiempo, observable y perceptible en forma directa) a cualquier instrumento de clasificación que se quiera usar.

Los ejercicios realizados en el estudio del DANE (2011) con el conjunto de datos provenientes del catastro permitieron seleccionar las variables, determinar su ponderación específica y su influencia en la definición de grupos homogéneos en el interior y heterogéneos respecto de los otros. Los resultados de diversas simulaciones y pruebas piloto mostraron que, por ejemplo, el área 
de construcción y el área de los predios no resultaba ser un dato diferenciador importante de los inmuebles, con el número de habitaciones (alcobas) o con la importancia de los garajes y parqueaderos. Todos estos cambios, que se reflejan en el peso que las variables establecen en los modelos puestos a prueba, han llevado a que muchas de las preconcepciones sobre las variables cambien.

De la misma manera y dado que distintos tipos de construcción con características similares se repiten por distintas localizaciones de la ciudad, la preconcepción sobre ciertos tipos de vivienda en lugares específicos ha dado lugar a comprender la variabilidad espacial de la vivienda a lo largo de toda la ciudad. Por su parte, los datos evidencian la importancia y capacidad discriminante que tienen la dotación, tipo, materiales y el mobiliario de baños y cocinas en la diferenciación de grupos. Estos van desde pequeños lugares funcionales pero pobremente dotados hasta grandes espacios con lujoso mobiliario y conservación en las localizaciones más exclusivas de la ciudad.

El estudio del DANE (2011) constituye un insumo fundamental a la hora de repensar la estratificación socioeconómica en Bogotá como fuente alternativa a la metodología vigente, lo que seguramente ofrecerá una clasificación distinta de los usuarios de servicios públicos domiciliarios acorde con la realidad material de su diferenciación. Incluso, genera luces para discutir sobre un eventual abandono de un modelo de estratificación y la administración de la política de focalización y manejo de subsidios y contribuciones por un sistema distinto que tenga como base directa de información las variables catastrales, y, entre ellas, las relativas a valores de suelo y valor de construcción, para las áreas no adscritas al régimen de propiedad horizontal, o valor integral, para aquellas donde prima dicho régimen. Estos escenarios fueron analizados de manera detallada en el capítulo 3, escrito por Bernal y Tejedor, donde se sustenta la importancia y potencialidad de la información catastral para captar la diferenciación urbana de Bogotá, en particular el uso del avalúo como insumo fundamental en la identificación de grupos socioeconómicos diferenciales. 